\title{
Iberoamérica, Ciudad Futura Ciudades sostenibles y medioambiente
}

\author{
Mara Sánchez Llorens
}

\section{RESUMEN}

La ciudad del futuro no es una página en blanco sino la ciudad existente y nuestra capacidad de transformarla. El siglo XXI va a ser el siglo de las ciudades y el futuro de la humanidad se desarrollará en ellas, su éxito radicará en ser sostenibles. Hay que repensar la ciudad: acercarnos a ella desde múltiples perspectivas y escalas, $y$ recuperar su lado humano.

El objetivo de este artículo es explorar algunas de las claves con las que enfrentarnos a la ciudad del futuro: alianzas entre la ciudad que ya existe y la ciudad que está por acontecer, alianzas entre lo natural y lo artificial que favorezcan ecosistemas urbanos adecuados para las sociedades urbanitas.

El texto, en primer lugar examina ciertas claves de entendimiento del propio concepto de ciudad sostenible a través de varias experiencias en Ciudad de México y, en segundo lugar, vislumbra nuevos modos de actuar en el diseño de la ciudad.

PALABRAS CLAVE: Sostenibilidad, diseño urbano participativo, presente histórico, reciclaje, reversibilidad, cuencas hídricas.

CLAVES ECONLIT: D74, N96, 021, Q25, R58.

Cómo citar este artículo / How to cite this article: SÁNCHEZ LLORENS, M. (2016): "Iberoamérica,

Ciudad Futura. Ciudades sostenibles y medioambiente", CIRIEC-España, Revista de Economía Pública, Social y Cooperativa, 87, 235-256.

Correspondencia: Mara Sánchez Llorens, profesora asociada, Universidad Nebrija y Universidad Pontificia de Salamanca en Madrid, E-mail: marasanchezllorens@gmail.com. 


\section{EXPANDED ABSTRACT}

\section{Ibero-America, Future City. Sustainable Cities and Environment}

"Cities are complex architecture works. Cities are the physical manifestation of the largest union of forces. Economic forces, social forces, environmental forces". (Urbanized, 2011)

Cities are explored as a critical component to transform human life, but when we talk about cities of the future, we mean that existing cities are not as blank pages but they are existing cites and our ability to transform them. We stand by this century will be the century of cities and the future of humanity will put into practice in them. Success of cities will lie in sustainability and environment. We must rethink the design of cities and approach to them from multiple perspectives and scales to recover their human side.

This paper proposes a critic exploration of some of the tools we can use to plan the city of the future to get alliances between current cities and future cities. The conjoined actions of these alliances configures a strategy that makes possible these future cities to remain essentially successful throughout time.

Thematically, the article begins with a dissertation about the Forum of Latin-American cities where aforementioned tools and other ideas to imagine the ways to change lbero- American cities into innovative cities were debated. Next, the text examines the concept of sustainable cities and environment through various experiences in Mexico City to deduce, secondly, new ways of acting in the urban-design of cities.

In order to sustain such argument, the paper proposes a review of cases to establish the viability of areas of collective use within both the geographic landscape and time. As a result, every concept is researched and put on an example of Mexico City.

The first example of the research studies an architectural ensemble, formed by a garden and two residential buildings called Isabel and Ermita buildings and they were designed by Juan Segura Gutierrez in the first thirties of the last century. Residents used to share the common spaces in a natural environment and there, they created social structures that brought together. At the present time, 
that garden doesn't exit and the two buildings are tumbledown but the neighbors remind the daily nature character there used to be there, and they demand to get back into that routine because it is part of their collective imagination.

Secondly, we will analyze a neighborhood called President Miguel Aleman designed with Modern Movement criteria by Mario Pani Darqui in 1949. This social condominium has public resources as swimming pool, tennis court, post office, laundromat and other installations. The film Elevator describes the evolution of the common spaces -inside and outside the residential buildings - and the current everyday life by elevator operator's testimony.

The last Mexican example consists in a territorial landscape project called Mexico: Future City by a collective of citizens, architects, engineers, geographers, biologists, sociologists and specializes in other disciplines that propose the complex interactions of multiple scales between territorial and anthropomorphic to recover the originally lake system of the metropolis, across four strategies: water, territory, energy and public spaces. This global project tries to generate a new system of lakes fed by the waste water that take place in the capital and seeks to give solution to the problem.

Methodologically, the research consisted on elaborating a database of good practices and selected sources from historical newspaper and periodicals library, social network, films and work in situ such as air-flies, interviews and inquiries related to the three Mexican examples and consequently synthesizes them in three deduced urban concepts. As a result, the research could obtain a timeline-poster to put on Latin American City.

The present experience is a way of communicating urban history, an applied example of the use of the timeline through the history of Latin American City, on an urban example: Mexico City. The suitability of this method of research is justified in the concurrency of urban, architectural and social details and in chronological order. The timeline offers an alternative to the academic model and to the inherited learning process, which identifies and exposes the transversal possible strategies to transform Ibero-American City.

The present article approaches three scales of the city, the first one is the scale of buildings, the second one relating to urban architectural ensembles and the last one in response to the territorial projects scale. The three scales must always improve and renew the public space and get the compactness of the city that has to offer to the users of urban projects that resolve their needs. We should not fear to turn urban highways into avenues or to re-formulate the shared spaces. Beside possessing the people, we will strain for realizing economically profitable practices, a solution to this problem is recycling -this suggests to transform our urban heritage, instead of demolish and build again. We will investigate the geography trying to revert the hurts caused to the environment. 
Our cities must take advantage of the resources and resign the culture of commanding discarded cards of an irrational way. Architecture and consistently City are inspired by nature that governs the and at the same time, nature offers architecture and city, the necessary instruments to give then harmony and form. The urban experimental spaces -as closed to the traffic streets during the weekends or the creation of new temporary topographies that are part of culture urban festivals- are contemporary urban actions that attract and assemble citizens. These kind of urban projects can transform cities as playful to support the festive environment during the accomplishment of an event and they can turn into a completely new way of working as urban designers by this methodology of large-scale work.

The Architecture of Future City point to urban models that take advantage of the nature resources, offer solutions that respect environment and propitiate spaces of conviviality and social harmony.

The main goal of this article is to discover the contemporary rules to put on practice in LatinAmerican Cities as Mexico City; therefore, the dissertation explores the urban evolution since the thirties to this moment through the study of three urban concepts: an idea of historical present, an idea of recovered city and the reversionary urban conversions. Metropolis will change this century into megalopolis ally with environment if they want to survive. Nature and citizens will be two of the highest players to encourage this sustainable revolution

KEY WORDS: Sustainability, Participatory Urban Design, Historical Present, Recycling, Reversibility, Water Basins. 


\section{1.- Introducción}

"La ciudad es una gran obra de arquitectura. Las ciudades son la manifestación física de las mayores fuerzas en juego. Fuerzas económicas, fuerzas sociales, fuerzas ambientales.»

(Hustwit, 2011)

Las ciudades crecieron de manera relativamente lenta en la época pre-griega y pre-romana -año 10 DC-, les tomó siglos llegar al millón de habitantes. En 1950 el 10\% de la población mundial pasó a vivir en ciudades. En el año 2010 lo hacía el 50\% de la población mundial y si el ritmo actual se mantiene, en 2050 lo hará el 75\% de la población mundial (Hustwit, 2011).

En marzo de 2015 se celebró en la ciudad mexicana de Veracruz el II Foro internacional Iberoamericano de Ciudades, Un encuentro entre ciudades, un contexto interdisciplinar que analizó la panorámica urbana iberoamericana y trató de generar modelos de desarrollo de ciudades, eficaces, inteligentes e inclusivas; es decir, ciudades sostenibles. Si aceptamos que el siglo XXI va a ser el siglo de las ciudades y que el futuro de la humanidad está en dichas urbes cuyo éxito radica en ser sostenibles: es necesario repensarlas. Nos enfrentamos, quizá, a un cambio de todo lo que sabemos y creemos sobre ciudades; posiblemente éstas funcionarán de manera totalmente diferente en las próximas décadas. Entendemos "sostenibilidad" como la capacidad de la humanidad de garantizar que satisface las necesidades actuales sin arriesgar la capacidad de que las generaciones futuras satisfagan sus propias necesidades (Comisión mundial de medioambiente y desarrollo, 1987). Cuando nos referimos al concepto de sostenibilidad lo hacemos desde los tres puntos de vista implícitos en el propio término: social, económico y medioambiental.

El Foro Iberoamericano de Ciudades nació en 2013. Celebró su primera edición en la capital peruana, Lima, con el objetivo de crear un espacio de intercambio entre los administradores públicos de la mayor parte de las ciudades de los países en los que se celebren las sucesivas ediciones y empresas que aporten soluciones tecnológicas y de gestión de entornos urbanos -un lugar para el debate y el intercambio, para el conocimiento, la formación y las nuevas estrategias urbanas-.

En el foro veracruzano señalado, algunas de las claves anotadas sobre el caso de estudio de las ciudades del país anfitrión profundizaron en ciertos datos dramáticos. Se advirtió de que el $80 \%$ de los mexicanos ya vive en ciudades (Rodríguez-Ortiz, 2015:125), siendo relevante que la reciente duplicación de la población se ha producido en un aumento de siete veces su superficie. A nivel mundial, cerca de un $54 \%$ de los habitantes de la Tierra vivimos en ciudades, estas ciudades ocupan alrededor del $3 \%$ de la superficie de la tierra y consumen las dos terceras partes de la energía y emiten el $80 \%$ de CO2, que es uno de los gases responsables del cambio climático (Fariña, 2015). 
La población total de México se ha multiplicado por cinco entre 1950 y 2000; en 2014 es de 119, 713,200 habitantes. El $80 \%$ vive en trescientas ochenta y cuatro ciudades. Previsiblemente en el año 2050 el $86 \%$ de la población de México, vivirá en ciudades. La población total de México se ha multiplicado por cinco entre 1950 y 2000; en 2014 es de 119, 713,200 habitantes. El 80\% vive en trescientas ochenta y cuatro ciudades (Rodríguez-Ortiz, 2015:134).

En el foro apuntado al comenzar este texto, se alertó de que algunos ciudadanos tardan más de dos horas en desplazarse a su puesto de trabajo, lo que significa que esas ciudades no funcionan. El análisis acusaba de políticas de vivienda inadecuadas y deterioro notable del parque habitacional $(41 \%)$, diagnosticaba que el suelo urbano estaba subutilizado, las infraestructuras eran obsoletas, el transporte insuficiente y la depredación del suelo era vertiginosa. Ante este panorama ¿qué modelo debería aplicarse en lo venidero? La respuesta fue contundente. El qué fue inmediato: las ciudades mexicanas del siglo XXI -podemos hacer extensible este dictamen a todas las latitudes- deberían ser ciudades sostenibles; el porqué se avaló con ingentes datos como los analizados previamente. Ahora bien, el cómo hacerlo fue lo que protagonizó el Foro, una respuesta múltiple y flexible, carente de certidumbres y verdaderamente compleja.

"Las ciudades, como los sueños, están hechas de deseos y temores; aunque el hilo de su discurso sea secreto, sus normas son absurdas, sus perspectivas engañosas y todas esconden siempre algo más" (Pieterse, 2008:7). A pesar de esta afirmación sobre lo absurdo de las normas en las ciudades, estas palabras no hacen sino hablar de la riqueza de lo urbano, como lo es la propia riqueza del ser humano -que precisamente es social de manera esencial- y de la riqueza y diversidad de las sociedades. La primera afirmación importante que podemos hacer es la necesidad de recuperar el lado humano de las ciudades.

Las ciudades son ecosistemas donde predomina la especie humana. Tienen gran dependencia del exterior porque en ellas no suele haber producción primaria, el consumo de energía es muy superior a la disponibilidad local de energías y la producción de desechos y de contaminación es muy elevada y debe ser expulsada fuera del entorno urbano. Tienen una gran incidencia sobre los ecosistemas de los que se nutre y a los que destina sus residuos. Este impacto se denomina huella ecológica de la ciudad. Dado que las ciudades constituyen la forma dominante de asentamiento humano y que nos encaminamos hacia un proceso de urbanización espectacular a nivel mundial, es preciso conocer cuál es la configuración y el metabolismo de los ecosistemas urbanos (Garrido, 2005).

Las ciudades son la expresión de sociedades diversas y en la actualidad, como ha sucedido casi siempre en la historia, muchas de estas sociedades se encuentran rotas, por ello el espacio público se vuelve simbólico y probablemente allí encontremos el cómo conseguir que las ciudades sean sostenibles, cuestión planteada inicialmente. La arquitectura y por ende, la ciudad, se encuentra entre el servicio público y la poesía. Una segunda afirmación importante es la necesidad de acercarnos y ver las ciudades desde múltiples perspectivas y escalas. 
La relación más intensa entre la ciudad y la sociedad se produce en las diferentes formas de espacio público: lugares de encuentro e intercambio de ideas. La ciudad contemporánea ha de reinterpretar el espacio público y materializarlo a través de planos colectivos y manipulables por los ciudadanos. Las tecnologías que conocemos en este momento también forman parte de esta nueva forma de espacio imantado, que es el espacio público (Sánchez, 2012:63).

Precisamente, lo que nos atrae de las ciudades es la oportunidad de dichos encuentros (Hustwit, 2011) y toda la magia que implica saber que podrás empezar aquí, terminar allí, volver, pero que algo inesperado sucederá en el camino y descubrirás algo; asimismo, la ciudad es el espacio encargado de conservar la naturaleza o identidad cultural. Esta manera de entender la ciudad es simultánea a la idea de que las ciudades son empíricamente los medios de innovación tecnológica y empresarial más importantes.

El siglo XX tuvo un modelo categórico: la ciudad debía ser moderna. Moderna o sostenible son dos objetivos abocados al fracaso si no se realiza como esfuerzo colectivo. La ciudad es más que un espacio físico, es un modelo de sociedad que permite el éxito de la diversidad, es un sistema global de pensamiento en el que lo importante es la gente.

Las mejores soluciones serán los mejores acuerdos y compromisos, no los mejores argumentos. Esta sería una tercera afirmación importante en el presente artículo.

Una vez planteadas estas tres premisas, el objetivo es explorar algunas de las claves con las que enfrentarnos a la ciudad del futuro, en una alianza entre la ciudad que ya existe y la ciudad que está por acontecer, una alianza entre lo natural y lo artificial. Proponemos acercar esas diversas miradas, partiendo de una propuesta importante: la ciudad del futuro no es una página en blanco sino la ciudad existente y nuestra capacidad de transformarla.

El texto en primer lugar examina ciertas claves de entendimiento del propio concepto de ciudad sostenible a través de varias experiencias de Ciudad de México que visualizan la ciudad desde escalas diversas y en segundo lugar, vislumbra nuevos modos de actuar en el diseño urbano. La ciudad contemporánea tiene retos para los nuevos modos de habitar, fluir, encontrar, atravesar, negociar 0 colaborar. Es decir, la ciudad contemporánea demanda nuevos modos de ser "moderno" (Serapião, 2013:18-122). 


\section{2.- Una idea de presente histórico}

"Las ciudades han hecho lo mismo durante tres, cuatro, cinco mil años» (Hustwit, 2011).

Cuando caminamos por una ciudad, todo lo que vemos fue pensado ¿Quién tiene permiso para dar forma a nuestras ciudades? ¿quiénes son los que han hecho lo mismo durante miles de años? La ciudad es una empresa colectiva en la que un grupo muti-disciplinario de gente ha trabajado para un mismo proyecto desde diferentes perspectivas, con diferentes intenciones y roles diversos. Arquitectos, empresarios y vecinos participan para crear hábitats humanos. Esta cooperación debería realizarse de manera equilibrada y participativa, siendo el espacio público el componente más importante.

La ciudad es un «lugar en el que confluyen de manera histórica las muchedumbres, el dinero, los bienes y a pesar de enfrentarnos a un proceso de cambio real definido como "era de la información", la importancia de las ciudades, queda redefinida por las condiciones en que se desarrolla "la nueva economía", centrada a partir de las transformaciones tecnológicas y organizativas» (Castells, 2001:208).

No podemos desperdiciar la oportunidad que nos brindan estos años de dificultad y aparente inmovilidad para pararnos a reflexionar sobre qué queremos aprender de los errores cometidos en nuestras ciudades y estudiar las posibilidades de las que disponemos para hacerlo mejor en un futuro siempre perteneciente a la contemporaneidad. Esta «experiencia del tiempo visto como presente es aquello que todavía está vivo y esa clase de presente nos ayuda a no caer en la trampa del futuro. Nuestra tarea es forjar otro presente verdadero, y para eso es necesaria la capacidad de entender históricamente el pasado y saber distinguir lo que servirá para las nuevas situaciones que hoy se nos presentan» (Bo Bardi, 1990:104).

Antes de participar en el Foro de Ciudades veracruzano con el que iniciábamos este texto, la estancia previa en Ciudad de México nos permitió revisitar algunos espacios recuperados del centro histórico como la peatonal calle Regina en la que las parcelas vacías dieron paso a pequeños parques urbanos; y experimentar de primera mano los cambios de la capital azteca de la última década, como la incorporación de la bicicleta. Fue la oportunidad de explorar varios ejemplos de intervención urbana en los que el concepto de presente histórico tomaba pleno sentido. Uno de aquellos espacios visitados tenía un especial interés añadido por haberse debatido recientemente en la opinión pública y en la Academia, acerca de su estado de abandono; se trata de un ejemplo extraordinario de la arquitectura decó mexicana -arquitectura realizada durante el periodo llamado Maximato, correspondiente a los años 1928-1934-, el conjunto arquitectónico Isabel-Ermita. 
En las primeras décadas del siglo XX, Ciudad de México desarrolló ejemplos arquitectónicos significativos acompañados por un aspecto único en el mundo, la síntesis de las artes manifestada a través de los murales públicos que fueron experiencias innovadoras y participativas de arquitectos, artistas, gestores y usuarios; y cuyo resultado fue una integración paisajística, urbana o no urbana, de la historia que acompañó, además, el crecimiento de la capital de entonces; creándose una suerte de circuito entre el centro histórico y la ciudad universitaria de los años cincuenta. Conjuntamente, en esta misma ciudad se desarrollaron complejos multifamiliares que emulaban retos arquitectónicos del eje Estados Unidos-Europa.

En la colonia suroccidental de Ciudad de México, Tacubaya, con un carácter de área de descanso por su clima agradable, agua y vegetación; el arquitecto capitalino Juan Segura Gutiérrez -con el mecenazgo de la Fundación Mier y Pesado- desarrolló entre los años 1928 y 1932 un conjunto urbano formado por edificios y un jardín. Tras la revolución mexicana de 1910 los primeros transportes públicos de la ciudad se planearon para que esta zona se incorporara a la ciudad.

Los edificios que tenían un programa mixto innovador de comercio-habitación y cine, fueron dos: el edificio Isabel (1928-29) y el edificio Ermita (1931-36) y se situaron en los extremos del predio. Las viviendas respondían a diversas tipologías -departamentos volcados al exterior en el perímetro y en altura, y viviendas unifamiliares en torno a pequeños jardines interiores. Los departamentos estaban destinados a parejas sin hijos o solteros, las viviendas unifamiliares estaban destinadas a familias con hijos- el uso comercial se situaba en la planta baja y el cine, cine Hipódromo Condesa, lo hacía en el edificio Ermita. Entre ambos edificios, como en el interior del Isabel, se conservaron unos jardines ancestrales (Katzman, 1964:176).

El edificio Ermita mide treinta y dos metros, cuenta con siete pisos y setenta y ocho departamentos. Su decoración Art Decó potenciaba la verticalidad. Se trataba de un edificio de renta que aspiraba a completar una política integral iniciada con la construcción del asilo Mier y Pesado, favorecida por la fundación privada del mismo nombre, que trataba de controlar la segregación residencial local y favorecer la integración social lugareña (Sabatini, 2007: 24). El complejo -que también trataba de ser un negocio- no escatimó recursos a la hora de ofrecer un programa en clave contemporánea que permitió que la gente humilde que lo habitaba pudiera disfrutar de la oferta de ocio más innovadora sin tener que desplazarse al centro, a pesar de que la llegada del tranvía fue una de las premisas de los Mier y Pesado para este triángulo de Tacubaya. Un testimonio de la época narra lo siguiente:

«Un sinito [cine pequeño] de barrio o al menos así lo veía yo. La gente iba a divertirse no importando las diferencias. De un lado venía la gente elegante, la gente bonita del lado de Revolución -allí había unas casas muy bonitas- y también llegaba gente normal.»

(Laboratorio Audiovisual de Investigación Social del Instituto Mora, 2008)

La escala del conjunto era ajena a la colonia (De Anda, 1990:118-124), hasta ahora rural y colindante con el Bosque de Chapultepec. El parque de Chapultepec es la principal reserva natural urbana 
de Ciudad de México con una extensión de 6,475,040 m². Sin embargo, el conjunto Ermita respetaba aquellas dimensiones que le hacen sentir cómodo al ser humano: «el ojo no puede dominar un área de más de cien por cien metros» (Hustwit, 2011). Los espacios públicos a veces no funcionan porque no se tiene en cuenta la relación de aquellos con los sentidos: qué tanto puedes ver y oír. «El objetivo de Segura era satisfacer no sólo las necesidades orgánicas del individuo sino también a favor de su capacidad de disfrute sensorial a través de la estética» (De Anda, 1990:121).

En el contexto que nos encontramos lo que también nos interesa destacar es la colaboración del arquitecto en el diseño de la ciudad. Segura propuso la propia urbanización de la cuadra, cediendo una franja a la travesía Revolución, lo que la convirtió en una avenida. Una sucesión de jardines intermedios que eran los vestigios del hermoso jardín de la casa en Tacubaya de la familia Mier y Celis / Pesado completaban el conjunto urbano.

El arquitecto fue sensible ante el presente histórico de la colonia que demandaba viviendas de renta. Tal y como promulgaba uno de los maestros de la arquitectura moderna, Le Corbusier, Segura hizo casas para hombres, sin pretender hacer nuevos hombres para las casas que él diseñaba; a la par, fue tremendamente respetuoso con el carácter y la necesidad de espacios abiertos para la convivencia que tradicionalmente disfrutaban de un ecosistema natural privilegiado por medio de los jardines diseñados y los ya existentes, que el arquitecto mexicano mantuvo para seguir ofreciendo a los usuarios el carácter verdadero de la colonia (Novo, 1947:65). La antigua ermita, que dio nombre al edificio, ahora era el edificio Ermita que se convirtió en un hito urbano y un reclamo publicitario vanguardista. El edificio se convirtió en un imán de gente admiradora de su belleza. Lo habitaron diversas celebridades ${ }^{1}$ y por todo esto, el barrio se reactivó.

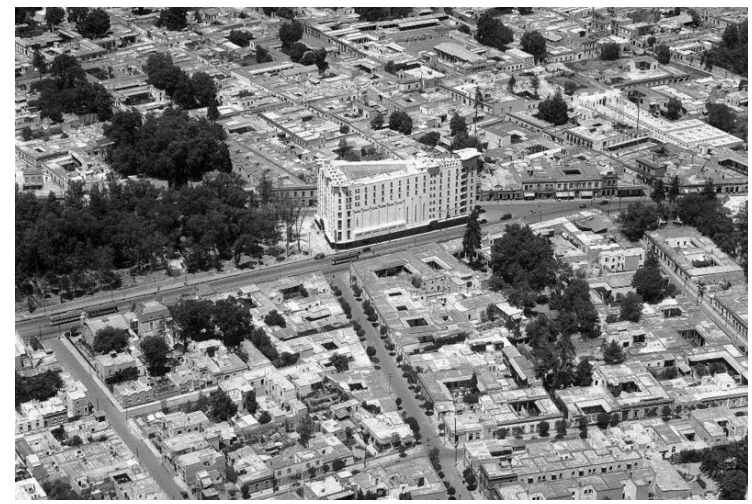

Triángulo Tacubaya, Finca de la familia Mier y Pesado, circa 1940. 
El debate que se producía en aquellos años sobre el rascacielos como respuesta a la necesidad de densificar la ciudad moderna, tuvo en esta geografía una respuesta con cierto espíritu ecológico que trascendió a partir de los años treinta a las colonias colindantes. Así arquitectos de reconocido prestigio como Enrique del Moral o Luis Barragán adquirieron terrenos cercanos en los que construyeron sus propias residencias y simultáneamente realizaron jardines secretos que milagrosamentetodavía hoy perviven. Los jardines del conjunto Isabel-Ermita desparecieron, sin embargo.

En julio de 2013 saltaba la alarma en el barrio. En 2014 la cuadra de los edificios de Segura, casi completa, había sido cercada y los establecimientos comerciales habían sido demolidos. El muro provisional se extendía hacia el cine situado en el Ermita, por lo que el mensaje era que también posiblemente sería demolido (Lázaro, 2014).

El México de los años treinta afirmó que "el funcionalismo está en los lugares en los que no se desperdicia ni un metro de terreno, ni el valor de un peso, ni un rayo de sol". El conjunto Isabel Ermita participó de este espíritu y debe conocerse y valorarse como tal, éste es un punto de partida por el que se aconseja la necesidad de actualizar el inventario del patrimonio arquitectónico. Una ciudad que no se quiere a sí misma, se degrada.

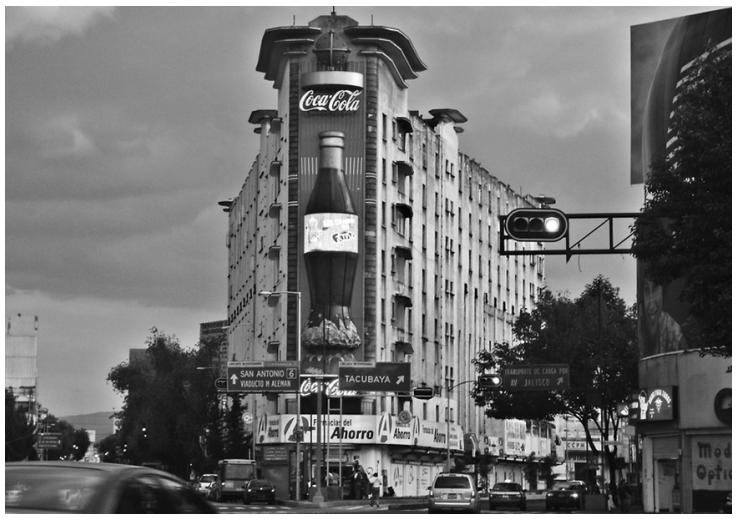

Edificio Ermita, Colonia Tacubaya, Ciudad de México, 2015 


\section{3.- Una contracultura al descarte}

En febrero de 2015 Glenn D. Lowry, actual director del neoyorkino MoMA, presentaba en el museo Rufino-Tamayo de Ciudad de México la exposición Latin America in Construction 1955-1980 que era una continuación de la exposición casi homónima de mediados del siglo pasado Latin American Architecture since 1945 y lanzaba la siguiente pregunta « ¿Dónde se ha construido la arquitectura más rica en los últimos cincuenta años?». La arquitectura y las actuaciones urbanas desarrolladas en Latinoamérica son desconocidas en muchos casos y es necesario aprender de la tradición de la ciudad existente para poder valorarla e iniciar su transformación, no debería permitirse que el patrimonio que no responde a los cánones internacionales o turístico se degrade y termine eliminándose. La arquitectura no debería convertirse en una escenografía urbana.

Regresemos al Foro lberoamericano de Ciudades con el que comenzábamos este artículo. Se plantearon otras claves para la intervención en la ciudad consolidada, lo que añade sentido a la idea previa sobre el presente histórico. Se proponía hacer lo siguiente con la ciudad existente: conocerla y valorarla, recorrerla en profundidad, inventariarla y proponer, planificar su nuevo carácter, recuperar vacíos, formalizar ciertos informalismos, si es indispensable empezar de nuevo, conservar, rehabilitar, restaurar; no olvidar incluir la naturaleza y reciclar lo existente. Estas claves admiten la oportunidad de experimentar la ciudad existente de una manera diferente.

En la pasada centuria, Iberoamérica, como otras muchas latitudes, desplegó su propia época del "desarrollismo" y aspiró a ser moderna, para ello debía romper con el pasado en nombre de dicha "modernidad". Las grandes capitales de aquellas décadas podían entenderse como dos ciudades simultáneas: la ciudad colonial y la nueva ciudad moderna. Las ambiciones latinoamericanas en el campo de los edificios públicos fueron representativas en el género de la vivienda.

La ciudad de ayer no funciona como la ciudad de hoy, la prioridad de las intervenciones muchas veces está en su localización; aquellos que intervenimos en la ciudad existente tenemos que generar las condiciones para que el flujo de gente en las ciudades suceda de la mejor manera.

El modelo occidental de desarrollo urbano ha quedado desacreditado tras la crisis financiera de 2008. De otro lado, ciudades iberoamericanas que han crecido en medio de crisis sistémicas se presentan como contra-modelos urbanos que ofrecen algo más a los ciudadanos que "máquinas productoras de riqueza" (Serapião, 2013:19) . 
La ciudad pasada no dudó en eliminar, destruir, hacer desaparecer las huellas de aquellas partes de la ciudad que no funcionaban en la era industrial. Concretamente el surgimiento de sitios postindustriales en las ciudades alrededor del mundo, ha llegado en los últimos treinta o cuarenta años y la gente no sabe qué hacer con ellos. Piensan que deberían ser eliminados. ¿Forma parte esa idea de la cultura del descarte imperante? Podemos pensar que la renovación de espacios en desuso para darles vida nuevamente, de una manera diferente, es un claro aprovechamiento de los recursos. Veíamos que la idea de presente histórico es una estrategia equilibrada entre pasado, presente y futuro, que no borra ni preserva a priori, sino que transforma, es decir, recicla y hace que la ciudad sea más sostenible. El Diccionario de la Real Academia Española define el término "reciclar" (en su edición de 2014) como "Someter un material usado a un proceso para que se pueda volver a utilizar». "Reciclar" forma parte del lenguaje básico de la ciudad del futuro.

Este tipo de intervenciones de reciclaje deberían basarse en el fortalecimiento de la identidad local y el sentido de pertenencia de sus habitantes con el fin principal de mejorar la calidad de vida de la población residente. (Etulain, 2013:212).

En la Bienal de Arquitectura de Venecia de 2012, su comisario, el arquitecto británico David Chipperfield, declaró: «Es el momento de pasar del "star system" a lo cotidiano»; es decir, una arquitectura amable y, por qué no, comprensible también para el profano. Se trata de aprovechar la crisis para recomponer la factura entre la arquitectura-espectáculo y la sociedad que la ha padecido.

Las narrativas de los pabellones de aquella edición de 2012 demostraron un interés notable por esta afirmación, destacando, el pabellón alemán que compiló ideas sobre cómo reducir, reusar y reciclar la arquitectura. Lidiar con las infraestructuras existentes se ha vuelto la tarea más importante a la que nos enfrentamos hoy los arquitectos. El pabellón germano realizó una completa re-evaluación de las estructuras en sí mismas y de las implicaciones sociales e históricas necesarias para mejorar el tejido urbano y alcanzar las metas climáticas. Estos conceptos también afectan a la ciudad, si ésta pretende ser sostenible.

En la colonia Valle, al sur Tacubaya donde se sitúa el conjunto Isabel-Ermita, y de manera simétrica a la avenida Insurgentes que estructura Ciudad de México de norte a sur; el arquitecto y urbanista Mario Pani Darqui diseñó y levantó en 1947, el primer conjunto habitacional de alta densidad de México y primer multifamiliar de América Latina, Unidad habitacional Presidente Miguel Alemán (Alva, 1999: 274) -conocido como Multifamiliar Alemán-. 


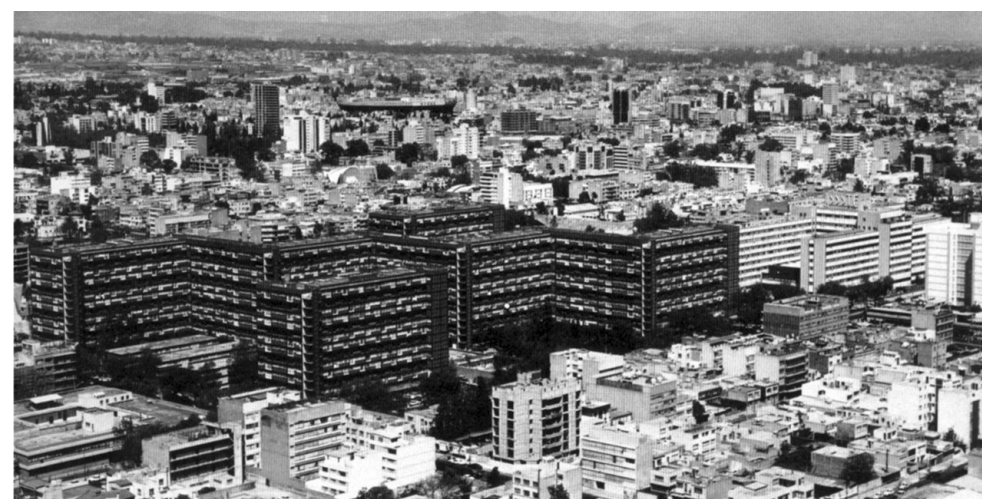

Vista aérea del Multifamiliar Alemán recién terminado.

Frente a las doscientas casitas que Pensiones Civiles, hoy llamado ISSSTE, encargó a Pani, el arquitecto construyó mil departamentos para trabajadores del Gobierno en seis edificios de trece pisos y seis menores de tres, rodeándolos de jardines, piscinas, espacios de esparcimiento, comercios, servicios sociales y escolares. El conjunto urbano también contaba con servicio postal, cocina y lavandería comunal dotadas de los electrodomésticos más modernos. Esta manera de diseñar la ciudad nos resultará a todos nosotros familiar, ya que la ciudad de la segunda mitad del siglo XX cuenta con ingentes ejemplos análogos.

"Obra gigantesca, moderna y única en el mundo, realizada por Ingenieros Civiles Asociados, ICA. La salud de los habitantes de esta ciudad se encuentra protegida por los servicios de una moderna unidad sanitaria. Aquí los padres si pueden estar tranquilos porque saben que sus hijos no serán víctimas de las calamidades de la ciudad moderna.» (Ortiz, 2012)

En 2012 el cineasta Adrián Ortiz presentaba el documental "Elevador" que cosecha éxitos desde entonces hasta la fecha de hoy. La cinta narra cómo el paso del tiempo ha convertido el multifamiliar Alemán en un espacio auto-administrado lo que ha generado un conflicto por la dificultad de gestionar los espacios colectivos que en origen fueron la "salvación" de los residentes, antes habitantes del centro de la ciudad y vivían en vecindades insalubres y anticuadas. Uno de los testimonios del largometraje apunta el siguiente diagnóstico:

«[El multifamiliar Alemán no funciona] Los departamentos [son] de dos pisos, hoy por hoy hay mucha gente de la tercera edad, las escaleras esas son un problema -en ese aspecto yo le decía a mi amigo Mario Pani que no pensó en que la gente envejece- Todos fuimos empleados del gobierno, órale que un veinte por ciento haya vendido, los demás son gente que estuvo aquí desde un principio, tenemos gente de noventa o más años. Aunque tengan familia, por problemas equis, porque también ellos no quieren irse de arrimados con los hijos, aun- 
que los hijos estén muy bien. Se quedan aquí, porque tienen su amor aquí, porque aquí han vivido, por lo que sea. El edificio está muy descuidado, muy sucio (...). Antes [el mantenimiento] lo pagaba el ISSSTE, ahora no. Nos dijeron, a partir de ahora va a ser tu propiedad. Ahora son once presidentes y no nos ponemos de acuerdo. Ya casi por aquí hay pocos jóvenes, pocos niños, eso se ha reflejado incluso en las escuelas.» (Ortiz, 2012)

Desde la actitud o contra-cultura al descarte ¿qué estrategias favorecerían la reintegración socioterritorial de este conjunto urbano? ¿Basta con conocerlo y valorarlo, es decir, incluirlo en catálogos que protegen el patrimonio urbano? Nosotros hemos de dar un paso más, el reciclaje no puede ser un trabajo de "fachadismo" sino una reactivación completa de los edificios y de su entorno con ciertas claves. Por un lado, aprovechar eficientemente los recursos naturales, minorizar el consumo energético, racionalizar el uso del patrimonio edilicio existente y disfrute de los recursos medioambientales. Por otro lado, trabajar con el programa funcional y ser creativos en su actualización trabajar con el edificio en el entorno urbano. La respuesta, la encontraremos en los habitantes del entorno urbano.

Hemos observado que la participación y las discusiones sirven a diversos fines. En primer lugar, aportan al planificador y a los habitantes información sobre necesidades y preferencias, lo cual es especialmente importante en situaciones en que están implicadas diversas culturas y el arquitecto sólo pertenece a una o es ajeno a ambas. En segundo lugar, es vital para el éxito del proyecto que el mayor número posible de habitantes comparta de buena gana y a sabiendas la responsabilidad de la creación y por ende de las consecuencias de los planes. En tercer lugar, es muy importante el aspecto pedagógico de dicho ejercicio, sobre todo con los menos privilegiados, que necesitan ejercitarse en el proceso del pensamiento abstracto, del análisis, de la resolución de los problemas y de la toma de decisiones si es que han de liberarse de su desventajosa situación y convertirse en ciudadanos auténticamente valiosos y valorizados, capaces de hacer contribuciones eficaces a una sociedad moderna, conquistando así el respeto por sí mismos (Sánchez, 2015:155).

Desde esta perspectiva los dos conjuntos urbanos Isabel-Ermita y Multifamiliar Alemán podrían convertirse en el desencadenante de la transformación sostenible de los barrios que los alojan. Los técnicos tenemos la responsabilidad de asegurar que económicamente la respuesta sea razonable y eficaz, debemos tratar de no destruir las estructuras sociales allí existentes, debemos ser flexibles además ante los nuevos posibles cambios para las generaciones futuras y para ello debemos contar con la participación: humana y del lugar. Debemos planificar una colaboración participativa específica para el lugar particular. Se trata de un sistema dialogado de creación, otras disciplinas como la sociología y la antropología nos enseñarán a incluir metodologías participativas en nuestra tarea. No es sólo cuestión de edificios sino de personas y de sus necesidades. El fin debe ser crear un diálogo entre la ciudad y los habitantes. 


\section{4.- Reversibilidad de los hechos urbanos}

Durante más de seis mil años los ciudadanos vivieron en las calles hasta que apareció el automóvil. En los años cincuenta el vehículo comenzó a tener un impacto real en las ciudades, por ejemplo la avenida Revolución o la avenida Insurgentes de Ciudad de México que alojan los multifamiliares Ermita y Alemán se crearon para este fin. El efecto de los autos en las ciudades fue ampliamente negativo porque esto afectó a la propia manera de diseñar la ciudad: es un problema global en el que la solución no es ampliar las avenidas ni crear segundos pisos, no, esto no resuelve los problemas de tráfico. La única salida es la restricción del uso del automóvil, para ello es necesario restringir el espacio de estacionamiento y devolverle ese espacio al peatón.

En la ciudad deben aliarse de nuevo los espacios públicos y la naturaleza. Es imprescindible que Iberoamérica rescate caminos y herramientas urbanas que convirtieron sus metrópolis en contextos diversos, cosmopolitas y pioneros, tal y como demostró la exposición ya apuntada Latin American Architecture since 1945 (Hitchcock, 1955:11-31).

Es necesario recorrer de nuevo y en profundidad la ciudad existente, descubrir la naturaleza que aún existe y favorecer una relación de ésta con las infraestructuras existentes. Es necesario que en la ciudad existente se analice y se actúe desde enfoques que prioricen el papel de la memoria colectiva del presente histórico, dentro del contexto más amplio de las transformaciones de las áreas metropolitanas: muchas podrán ser reversibles, y renovar el papel que desempeñaban en el pasado, sin convertir la ciudad existente en una representación imaginaria de la vida urbana tradicional frente a una expansión periférica deshumanizada y "desurbanizada" (Hiernaux-Nicolas, 1999:42).

En 1951 el arquitecto mexicano Alberto T. Arai expuso el Plan Texcoco en la comunicación «El hundimiento de la Ciudad de México y su posible solución urbanística», dentro del VIII Congreso Panamericano de Arquitectos celebrado en la metrópoli mexicana. En dicho plan se afirmaba que gran parte del cambio ambiental producido en la capital azteca era consecuencia directa de la significativa deshidratación producida por el ser humano en las tierras mexicas en las recientes centurias y que, en consecuencia, produjo la desecación del sistema lacustre originario. Ante tal diagnóstico, la respuesta ofrecida por Arai consistía en la reversibilidad de los acontecimientos: inundar de nuevo la metrópoli para que ésta recuperara su condición pantanosa.

De manera alarmante y desde aquellos años cincuenta, diversos colectivos de protección de la naturaleza han alertado al planeta -en congresos y publicaciones como el significativo Congreso organizado en Caracas en 1952 por la Unión Internacional de Protección de la Naturaleza (Bo Bardi, 1992: 15)- del peligro del desvío de los grandes cursos de agua y deforestaciones. 
En 1991, los arquitectos mexicanos Teodoro González de León y Alberto Kalach retomaron el Plan Texcoco de los años cincuenta y realizaron un profundo diagnóstico sobre lo acontecido en la ciudad lacustre. Desde 1997 desarrollaron un proyecto concreto, Vuelta a la ciudad lacustre, en el que propusieron recuperar los lagos que convivieron armoniosamente con los mexicas, y que la historia se había empeñado en agotar (González de León, 2011:14).

Este proyecto ansiaba ser un reencuentro de la ciudad de México y el agua de ríos y lagos desparecidos, ahora invisibles. Un reencuentro respetuoso con la condición histórica y cultural de los mismos: presente histórico. Un reencuentro, que cualificaba áreas de oportunidad existentes como respuesta a la demanda de nuevos espacios y necesidades públicos. Un reencuentro que era a la vez desencadenante del reciclaje del este de la ciudad.

Hasta ahora, la forma de abordar el problema planteado por la condición lacustre capitalina se había llevado a cabo por el camino erróneo, pensando que el lago es un accidente de la ciudad. Nada más lejos de la realidad, Ciudad de México es un evento propio del lago de Texcoco.

A través del colectivo México, Ciudad Futura se aunaron los esfuerzos de ingenieros, biólogos, urbanistas, estudiantes y sobre todo, ciudadanos, para recuperar la condición lacustre de la metrópoli mexicana, proponiendo a través de un manifiesto en la red y en las librerías de todo el mundo actuaciones de múltiples escalas entre territoriales y antropomórficas, a través de cuatro estrategias: agua, territorio, energía y espacios públicos. Este proyecto global trataba de generar un nuevo sistema de lagos alimentados por las aguas residuales que se producen en la capital y busca dar solución al problema presente de degradación del ecosistema natural de la Ciudad de México y en el que el hombre había sido causante -al menos parcialmente-. El colectivo apuntado trataba de comunicar a los mexicanos el funcionamiento del ecosistema natural prehispánico, las consecuencias de la posterior y continua desecación de los lagos y consensuar, entre todos y desde la democratización de los espacios públicos, cómo devolverle a la ciudad el ecosistema perdido tratando de reconducir con ello el propio crecimiento de la metrópolis (Kalach, 2012) y crear una tendencia hacia la autosuficiencia urbana del agua por medio de la transversalidad de todas las disciplinas que afectan a la ciudad y la investigación propositiva que se convierte en praxis real sin miedo a los largos plazos.

El proyecto México, Ciudad Futura tratará de responder y actuar desde una esfera geográfica, entendiendo que negociará con un continuo biológico, un vector cargado de energía, que no se puede segregar según el viejo binomio rural-urbano. Ciudad de México ya no es la ciudad lacustre de Tenochtitlan pero para sobrevivir, tampoco puede seguir siendo la metrópoli actual.

El lector puede deducir ahora si esta estrategia sostenible para el diseño de las ciudades del tercer milenio es posible con la implicación de todos. Nuestra respuesta es "Sí», es posible y es deseable. Al sobrevolar hoy la capital mexicana, uno contempla cómo aquel basurero que provocó un incremento de contaminación en el aire en los años cincuenta, se diluye; y en su lugar, el progresivo Parque Ecológico Texcoco recibe al visitante y reinventa el futuro de Ciudad de México. 
México, Ciudad futura no ha contado con todo el ánimo de los usuarios desde el principio de su concepción: no seríamos sinceros si no lo mencionáramos. Los profesionales exhibieron un resultado e hicieron partícipes a los ciudadanos después. Quizá por eso es necesario que la ciudad del futuro sea un éxito colectivo. Poco a poco, aunque sean pasos pequeños.

Entre los beneficios ya obtenidos con este proyecto urbanístico se encuentran mejoras en la calidad del aire, limitación de los hundimientos diferenciales del país, disponibilidad de agua potable y agua de riego para zonas agrícolas periféricas, aumento de áreas verdes, así como nuevos desarrollos urbanos que alojarán, en el mediano plazo, el crecimiento de la zona metropolitana de la Ciudad de México. El nuevo aeropuerto está situado allí y ya ha comenzado sus obras. Pero el mayor beneficio, del que ya disfruta Ciudad de México, es el de recuperar la esperanza por el fututo de su ciudad y en ello los ciudadanos ya son partícipes a través de múltiples medios: asociaciones vecinales, encuestas, encuentros y congresos. Esta participación no aspira a sustituir el trabajo de las múltiples disciplinas que han de trabajar por la ciudad para reorientar la forma urbana, el transporte, la energía y los recursos; sino que tratan de implicar al ciudadano para que éxito sea colectivo.

En la exposición del MoMA que hemos mencionado en el trascurso del texto se afirmaba que la ciudad desde los años cincuenta fue planificada "a vista de aeroplano" y la ciudad debe verse desde la perspectiva de la calle, como lo hizo Juan Segura en la colonia Tacubaya, que supo detectar la estructura social que ya existía allí funcionando muy bien. Las comunidades tenían costumbres diversas y él trató de darles un mejor espacio físico en el que seguir desarrollando su vida, poniendo los ojos de la gente y en la calle, lo que hizo del conjunto urbano un espacio más seguro y cómodo. Quizá ese es el mayor hallazgo que podemos compartir en estas líneas: la ciudad sostenible sólo es posible si se realiza "a pie de calle".

\section{5.- Conclusiones}

El futuro de la arquitectura apunta hacia modelos urbanos que aprovechen los recursos naturales, ofrezcan soluciones respetuosas con el medio ambiente y propicien espacios de convivencia.

Nos hemos acercado a tres escalas de la ciudad, la de los edificios, la de los conjuntos urbanos y la de los proyectos territoriales. Las tres escalas deben relacionarse siempre con el objetivo de mejorar y renovar el espacio público y procurar la compacidad de la ciudad ofreciéndole al usuario proyectos que resuelvan sus necesidades. No nos debe dar miedo convertir las autopistas urbanas en avenidas o reformular los espacios compartidos. 
Además de contar con la gente, nos esforzaremos por realizar prácticas económicamente rentables, una solución a este problema es, en muchas ocasiones, el reciclaje -esto implica transformar la mayoría de nuestro patrimonio urbano, en lugar de descartarlo a priori-, además, le preguntaremos a la geografía tratando de revertir los daños causados al medioambiente. Probablemente, como afirmábamos anteriormente, no importa tanto cómo sean la arquitectura sino la calidad del espacio público y su ubicación.

Parafraseando a Aldo van Eyck, una ciudad sostenible es aquella urbe en la que la suma del esfuerzo de sus gentes hace que quieran quedarse a vivir en ellas para poder caminar, conversar, jugar; el crecimiento económico más prodigioso, si no van acompañados por un auténtico progreso social y moral, se vuelven en definitiva contra el hombre (Francisco, 2015:4). Nuestras ciudades deben aprovechar los recursos y renunciar a la cultura de descarte imperante de manera irracional. La arquitectura y consecuentemente la ciudad, se inspiró en la naturaleza que la gobierna y que al mismo tiempo le ofrece los instrumentos necesarios para darle forma y armonía.

Las estadísticas y los datos sobre el futuro de las ciudades le hacen a uno ser tremendamente pesimista pero la historia demuestra «que se necesita un pequeño grupo de innovadores, un grupo pequeño de gente que puede demostrar cómo hacer las cosas de manera diferente y una vez en marcha, el cambio puede darse de manera realmente rápida» (Pieterse, 2008:18).

Una visión arquitectónica del futuro evalúa, por un lado y a través de diversas herramientas que coordinan múltiples indicadores que interactúan entre sí -como LEED, BREEAM, CASBEE o la Certificación del Urbanismo Ecosistémico del español Salvador Rueda- la calidad de lo que ya ha sido construido y plantea proyectos de renovación de las ciudades. Por otro lado, la apuntada visión arquitectónica del futuro de la ciudad investiga de manera cualitativa la experiencia de los propios usuarios.

Los espacios urbanos experimentales como las calles cerradas al tráfico durante los fines de semana o la creación de nuevas topografías, son actuaciones que atraen y reúnen a los ciudadanos. Estos proyectos, pueden comenzar como ideas lúdicas para apoyar el ambiente festivo durante la realización de un evento y convertirse en una forma completamente nueva de trabajar con el diseño urbano; una metodología de trabajo a gran escala. Sus diferentes configuraciones y posibilidades espaciales pueden ser comprobadas en terreno, para luego ser replicadas o aplicadas de forma permanente.

Siempre he admirado el fenómeno de las ciudades y creo que es posible ser optimista de cara a las ciudades en nuestra época. 


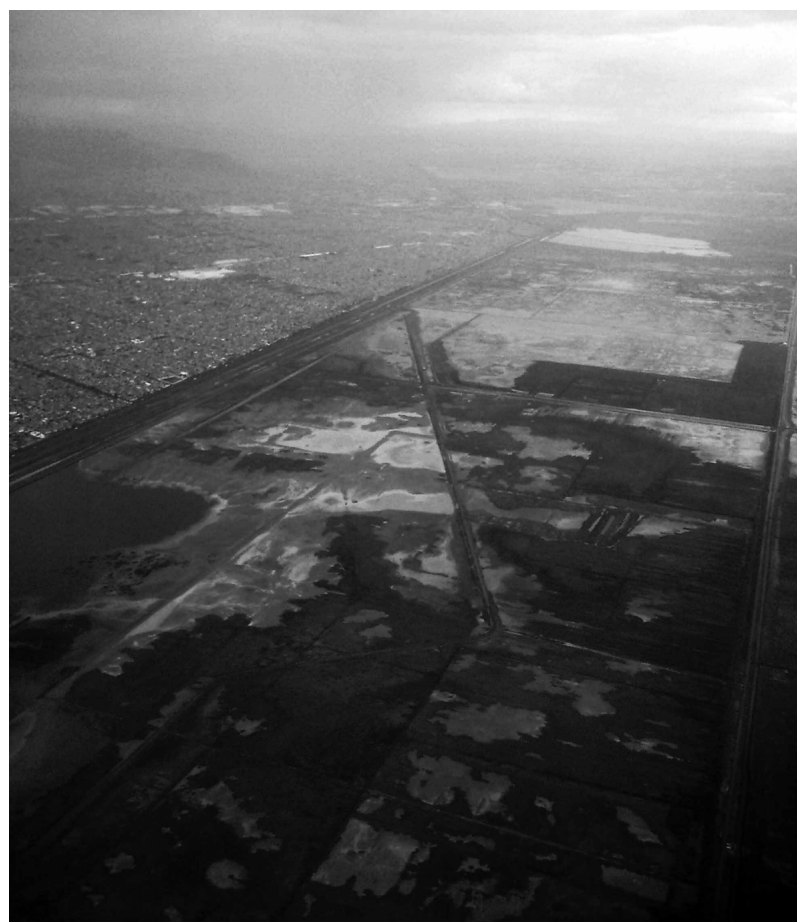

Estado actual del Parque Texcoco y la recuperación del ecosistema lacustre. Vista aérea tomada en marzo del año 2015.

\section{Bibliografía}

ALVA, E. (1999): Guía de Arquitectura de Ciudad de México, Departamento de Publicaciones de la Secretaría General de Planificación de la Junta de Andalucía, Sevilla.

AUSUBEL, J.H. (1996): "The Liberation of the Environment", Deadalus, 125, 1-17.

BO BARDI, L. (1990): "Uma aula de arquitetura”, Projeto, 133, 103-108.

BO BARDI, L. (1992): Contribução Propedêutica ao Ensino da Teoria da Arquitetura (1957), Instituto Lina Bo \& P.M. Bardi, São Paulo.

CASTELLS, M. (2001): "La ciudad de la nueva economía", Papeles de Población, 27, 207-221. 
DE ANDA, E. (1990): La arquitectura de la revolución mexicana, Universidad Nacional Autónoma de México, México, 118-124.

ETULAIN, J.C. \& GONZÁLEZ, A. (2013): "Rupturas y reintegraciones socio-territoriales en la intervención de los centros históricos latinoamericanos". En: VII Congreso de Medio Ambiente de la Asociación de Universidades Grupo Montevideo, Universidad Nacional de La Plata, La Plata.

FORSYTE, J. (2015): The Poetics of Development, Velocity Filmworks, Nueva York.

FRANCISCO (2015): Carta Encíclica Laudato Si sobre el cuidado de la casa común.

GRUZINSKI, S. (2000): El pensamiento mestizo, Paidós Ibérica, Barcelona.

HITCHCOCK, H-R. (1955): Latin American Architecture Since 1945, The Museum of Modern Art, Nueva York.

HIERNAUX-NICOLAS, D. (1999): Los senderos del cambio. Tecnología, sociedad y territorio en el umbral del siglo XXI, Plaza y Valdés, México D.F.

KATZMAN, I. (1964): La arquitectura contemporánea mexicana precedentes y desarrollo, INAH-SEP, México.

NOVO, C. (1947): Nueva grandeza mexicana, FCE, Ciudad de México.

PIETERSE, E. (2008): City Futures: Confronting the Crisis of Urban Development, Zed Books, Cape Town.

RODRÍGUEZ-ORTIZ, A.Ma (2015): "Universo de ciudades emergentes". En: Desarrollo urbano y Vivienda en Latinoamérica, Banco Interamericano de Desarrollo, Washington.

RUEDA, S. (2012): Guía metodológica para los sistemas de auditoría, certificación o acreditación de la calidad y sostenibilidad en el medio urbano, Departamento de Publicaciones del Ministerio de Fomento del Gobierno de España, Madrid.

SÁNCHEZ, M. (2012): Arquitectura y sociedad, Fundación Pablo VI, Madrid.

SÁNCHEZ, M. (2014): "Colectivo México Ciudad Futura. Reversibilidad antropógena de los hechos urbanos", Rita, 2, 84-91.

SERAPIÃO, F. (2013): "X Bienal de Arquitetura de São Paulo, Monolito, 17, 18-122. 


\section{Consultas realizadas en internet}

FARIÑA, J. (2015): "Una ciudad más próxima", El País, online <www.internacional.elpais.com> [última consulta 31/08/2015].

GARRIDO, F.J. (2005): "El ecosistema urbano". En: Gloobalhoy, 6, online <www.gloobal.net>[última consulta 28/08/2015].

KALACH, A. (2012): "México. Ciudad futura", online <www.kalach.com/proyectos/ciudad-futura/ciudadfutura.html>.

EL EDIFICIO ERMITA: BREVE HISTORIA (2014): online, Edificios de México <www.edemx.com>.

LABORATORIO AUDIOVISUAL DE INVESTIGACIÓN SOCIAL DEL INSTITUTO MORA (2008): Triángulo de Tacubaya, online <www.lais.mora.edu.mx/publicaciones/index.php/topics/single/4>

LÁZARO, C. (2014): El Cine Ermita de Tacubaya hacia la demolición, online <www.lamendigapolitica.com/blog/?p=21478> [última consulta 19/08/2015].

ONU-HABITAT (2012): Estado de las ciudades de América latina y el Caribe. Rumbo a una nueva transición urbana, online <www.unhabitat.org > [última consulta 20/08/2015].

SABATINI, F. (2007): "Integración social urbana en la nueva política habitacional", Dirección de asuntos públicos, 7, online < www.minvu.cl> [última consulta 31/08/2015].

UNITED NATIONS (2014): "World Urbanization Prospects", Final Report, online <http://esa.un.org/unpd/wup/FinalReport/WUP2014-Report.pdf> [última consulta 24/08/2015].

URBATHEL (2013-2015): Foro Iberoamericano de Ciudades: <www.foroiberoamericanodeciudades.org> [última consulta 31/08/2015].

\section{Largometrajes}

ORTIZ, A. (2012): Elevador, Documental, Sardina Films (72 minutos).

HUSTWIT, G. (2011): Urbanized, Documental, Gary Hustwit Films (85 minutos). 